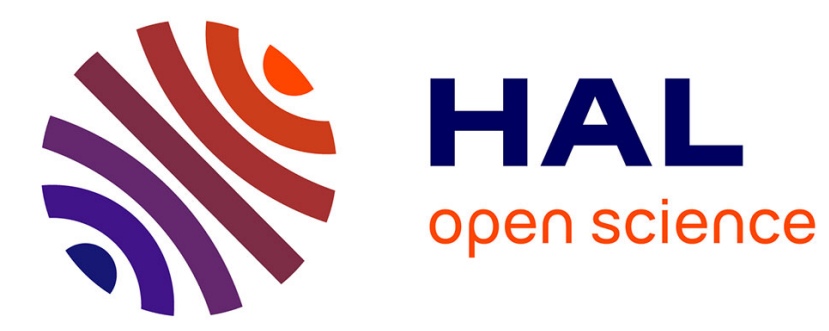

\title{
Modélisation du processus de sédimentation-consolidation des vases estuariennes: approche continue unidimensionnelle
}

S. Gallois, A. Alexis, P. Thomas

\section{> To cite this version:}

S. Gallois, A. Alexis, P. Thomas. Modélisation du processus de sédimentation-consolidation des vases estuariennes: approche continue unidimensionnelle. Bulletin of Engineering Geology and the Environment, 1999, 57 (3), pp.311 - 318. 10.1007/s100640050050 . hal-01007072

\section{HAL Id: hal-01007072 \\ https://hal.science/hal-01007072}

Submitted on 23 Jan 2017

HAL is a multi-disciplinary open access archive for the deposit and dissemination of scientific research documents, whether they are published or not. The documents may come from teaching and research institutions in France or abroad, or from public or private research centers.
L'archive ouverte pluridisciplinaire HAL, est destinée au dépôt et à la diffusion de documents scientifiques de niveau recherche, publiés ou non, émanant des établissements d'enseignement et de recherche français ou étrangers, des laboratoires publics ou privés.

\section{(c)(1)}

Distributed under a Creative Commons Attribution| 4.0 International License 


\title{
Modélisation du processus de sédimentation-consolidation des vases estuariennes: approche continue unidimensionnelle
}

\author{
Modelling the process of sedimentation-consolidation \\ for estuarine sediments: a continuing uni-dimensional \\ approach
}

S. Gallois · A. Alexis · P. Thomas

S. Gallois $(\square) \cdot$ A. Alexis · P. Thomas

Laboratoire de Génie Civil, Institut Universitaire de

Technologie, BP 420, F-44606 Saint-Nazaire Cedex, France

e-mail: gallois@iutsn.univ-nantes.fr, Fax: +33-2-40-17-81-60

\begin{abstract}
Résumé L'étude de la sédimentation et de la consolidation des sédiments cohésifs présente un grand intérêt scientifique par son aspect interdisciplinaire, à la frontière hydraulique-géotechnique et en raison de l'importance de ses applications à la gestion des zones cotières. Nous proposons d'associer les points de vue hydraulique et géotechnique en vue d'établir un modèle continu du processus de sédimentationconsolidation basé sur les équations de conservation de deux phases compressibles. Ce modèle, général et évolutif, permet d'envisager la prise en compte de phénomènes négligés jusqu'alors: fluage, floculation, écoulements non-darciens, ... Il constitue donc un élément fondamental pour une modélisation globale et précise de la dynamique sédimentaire en estuaire.
\end{abstract}

Mots clés Sédimentation - Consolidation - Vase · Modélisation

\footnotetext{
Abstract Nowadays numerical models tend to replace physical models for the study of sediment transport processes in fluvial or marine environments. The variability of both the materials and the environments make the processes complex in areas of major economic importance such as estuaries. The representativeness of current models depends much on the evaluation of the material which is deposited and/or re-suspended, hence the modelling
}

of the deposit and consolidation phenomena. The paper proposes a unidimensional analysis of the sedimentation-consolidation process based on a continuous action and taking account of the complexity of the sedimentary materials. The distinction between sedimentation (the fall of isolated grains in a fluid medium) and consolidation (compression of a solid deposit by evacuation of pore water) is difficult to determine in the case of muds. In muds, particles frequently interact (for example in flocculation and deflocculation) although the grains remain separated by a film of water at a microscopic scale. In the 1980s and 1990s, several authors attempted to unify the two phenomena. This paper reviews the most significant works on sedimentation and consolidation and discusses their limitations, noting that there is always a boundary between the two processes. The model proposed in this paper assumes a continuity of the sedimentation-consolidation process which remains compatible with previous theories. It is based on wide assumptions, postulating the effective stress can be time dependent and the infiltration velocity is not necessarily governed by Darcy's law. A system of equations is derived which are valid for a polyphasic medium (solid, liquid, gas) and where the solid phase can be composed of isolated grains and aggregates. State equations, equations of mass conservation and equations of mechanical equilibrium are completed by laws derived from the experimental approaches described in this paper or from those of other authors. The model allows the introduction of various shapes of constitutive laws, incorporating 
time in the effective stress function (taking account of creep phenomena) and a filtration velocity which does not follow Darcy's law (notably for the very low hydraulic gradients). This new model is applicable to a great number of sedimentation and/or consolidation cases, e.g.

- Under water sedimentation-consolidation cyclic load (swell, tide, etc.).

- Consolidation of mud in ground deposits.

- Consolidation of soft clay layers.

The model has been validated by means of the sedimentation-consolidation simulation tests as undertaken by Been (1981). Despite the use of simple constitutive laws, the model gives a theoretical evolution of density profiles which is very similar to the experimental one for the same initial conditions. This model of the transition between sedimentation and consolidation has a wide field of application, including civil engineering, medicine, the food industry, chemical engineering and ecology.

Key words Sedimentation $\cdot$ Consolidation $\cdot$ Mud · Modelling

\section{Introduction}

Les phénomènes d'érosion et de dépôt des sédiments fins sont au cœur des préoccupations des ingénieurs pour tous les projets d'aménagements fluviaux, estuariens ou maritimes. En effet, toute modification de la morphologie des rives, des côtes ou même des fonds, altère localement les phénomènes de transport sédimentaire. Ces modifications peuvent avoir des conséquences néfastes pour les constructions et pour l'environnement: déstabilisation de rives ou d'ouvrages, comblement de passes, de lagunes ...

Pour prévoir l'impact d'un aménagement, on a souvent recours à des modèles physiques extrêmement coûteux. Des modèles numériques adaptés permettraient de généraliser ces études à tous les types de sites et apporteraient des informations sur certains phénomènes mal connus comme les bouchons vaseux en estuaire ou la dispersion des sédiments dragués.

De nombreux logiciels (TELEMAC, élaboré par le Laboratoire national d'hydraulique, LTFATE, de l'US army corps of engineers, REJPAR, du laboratoire INRS océanologie de l'université de Québec) proposent une modélisation du dépôt et de la remise en suspension de sédiments. Ces phénomènes étant généralement cycliques (causés par la houle ou la marée), la qualité de la modélisation dépendra de la précision de l'évaluation de la masse de sédiments mobilisable après chaque période de dépôt et consolidation. Notre analyse de la phase sédimentation-dépôt- consolidation d'un sédiment estuarien, autorisera la prise en compte de la grande complexité de ces matériaux, ainsi que de leur variabilité spatio-temporelle.

\section{Particularité du phénomène en environnement estuarien}

La sédimentation est définie comme le processus de chute d'éléments solides (grains, flocons, agrégats ...) dans un liquide, sous l'action de la pesanteur et des forces de frottement dues à la viscosité du liquide. La consolidation est le phénomène de tassement d'un dépôt particulaire par évacuation du liquide interstitiel sous l'action de la pression due au poids propre des particules et/ou à un chargement extérieur. Les deux phénomènes ont ainsi été fréquemment dissociés selon plusieurs critères:

1. En sédimentation les grains ou agrégats sont éloignés les uns des autres, tandis que lors de la consolidation les grains sont très voisins, voire en contact.

2. En sédimentation, les contraintes effectives (interactions directes entre grains) sont considérées comme nulles.

3. En sédimentation les grains chutent dans un fluide immobile tandis qu'en consolidation le fluide circule dans un squelette fixe.

Dans le cas des vases fluviales, marines, ou estuariennes, matériaux de grande hétérogénéité caractérisés par une proportion importante de particules fines argileuses et organiques, ces distinctions semblent a priori mal adaptées. En effet:

Les couches d'eau adsorbée et d'eau pelliculaire fixées à la surface des plaquettes argileuses, rendent les contacts directs entre solides très improbables, même dans les matériaux fortement consolidés:

1. L'observation de la formation et de l'éclatement de flocons au cours de la sédimentation peut laisser supposer l'existence d'interactions à distance entre grains pouvant être assimilées à des contraintes effectives.

2. Pour des concentrations intermédiaires, les vitesses absolues des grains et du fluide peuvent être du même ordre et il n'est alors pas possible de négliger l'une ou l'autre.

En conséquence, pour des sédiments cohésifs fins, il apparaît que les phénomènes de sédimentation et de consolidation constituent un processus physique continu dans l'espace et dans le temps. C'est pourquoi nous nous attachons, dans cet article, à établir une modélisation continue de lasédimentation-consolidation reposant sur des équations définies et continues sur l'ensemble du domaine spatio-temporel considéré (Fig. 1).

Par ailleurs, la salinité de l'eau et la présence de matières organiques au sein du matériau ont une influence importante sur le comportement bio-chimique des sédiments. Sans préjuger de cette influence, nous estimons qu'elle ne peut, généralement, être négligée. Notre modèle doit donc être capable de prendre en compte les phénomènes de 


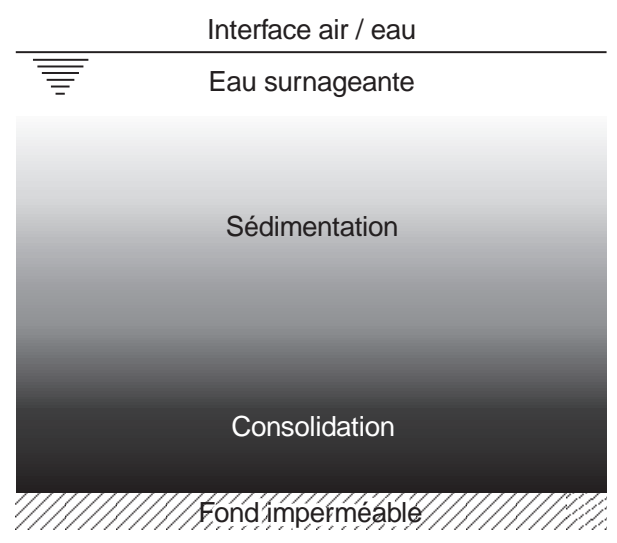

Fig. 1

Représentation schématique du domaine d'étude et de ses frontières inférieure et supérieure

Fig. 1

Schematization of studied field and its lower and upper boundaries

floculation-défloculation ainsi que la présence de gaz, notamment dans le milieu consolidé.

Bien que consacré principalement au comportement des sédiments fins ou vases, la portée de notre travail dépasse largement le domaine du génie civil pour s'étendre à l'agro-alimentaire, au génie chimique, à la médecine, à l'écologie ...

Dans un premier temps nous proposons une revue des principales modélisations antérieures, en sédimentation et en consolidation, ainsi l'analyse de leur adéquation à la sédimentation-consolidation. Nous explicitons ensuite une à une les équations constituant notre modèle et les hypothèses faites. Enfin nous étudions les possibilités d'application de cette théorie.

\section{Théories de la sédimentation et de la consolidation}

Les phénomènes de sédimentation et de consolidation, considérés jusqu'alors comme distincts, ont été étudiés respectivement par des hydrauliciens et hydro-sédimentologues et par des géotechniciens et mécaniciens des sols. Une synthèse des différentes approches proposées dans chaque spécialité ayant été effectuée par Alexis et al. (1993), nous détaillons seulement ici les travaux les plus marquants en étudiant particulièrement leur éventuelle aptitude à modéliser l'ensemble du processus de sédimentation-consolidation.

\section{Sédimentation}

Les travaux de Stokes (vers 1850) posent les fondements de l'étude de la sédimentation par le calcul de la vitesse de chute d'une particule sphérique isolée dans un fluide newtonien. Les hypothèses sur les particules empêchent l'application de cette théorie à la chute de sédiments fins dans l'eau (forme des plaquettes argileuses, concentrations importantes).

La théorie de Kynch (1952) admet que l'écoulement peutêtre perturbé par la proximité des grains (notion de «chute entravée») et repose sur l'hypothèse que, pour un matériau donné, la vitesse de chute des grains ne dépend que de leur concentration locale. Cette théorie conduit à une équation (1) reliant la vitesse absolue des grains solides, $\mathbf{v}_{\mathbf{s}}$, à leur concentration pondérale (ou poids volumique sec) $\gamma_{\mathrm{d}}$, en fonction du temps $\mathrm{t}$ et de la cote $\mathrm{x}$ (orientée vers le haut):

$\frac{\partial \gamma_{\mathrm{d}}}{\partial \mathrm{t}}+\frac{\mathrm{d}\left(\gamma_{\mathrm{d}} \cdot \mathrm{v}_{\mathrm{s}}\right)}{\mathrm{d} \gamma_{\mathrm{d}}} \cdot \frac{\partial \gamma_{\mathrm{d}}}{\partial \mathrm{x}}=0$

L'expression de la vitesse de chute synthétise ainsi toute la complexité du processus. La validité de cette théorie, apparemment simple, dépend donc entièrement du type de loi choisi pour la vitesse de chute des grains, $\mathrm{v}_{\mathrm{s}}\left(\gamma_{\mathrm{d}}\right)$, et de la représentativité de cette loi. Des lois relativement simples (Toorman 1992) permettent une modélisation assez fidèle de la phase de sédimentation.

\section{Consolidation}

L'étude de la consolidation a été l'apanage des mécaniciens des sols depuis les premiers travaux de Terzaghi (vers 1925). La théorie de Terzaghi (1942) fait apparaître deux lois de comportement caractéristiques du matériau décrivant les variations des contraintes effectives et de la perméabilité. Les hypothèses proposées sur ces lois permettent la résolution de l'équation de consolidation unidimensionnelle (ou équation de dissipation des surpressions interstitielles) mais en limitent l'application au cas des déformations infinitésimales (c'est à dire petites devant l'épaisseur du dépôt).

Gibson et al. (1967) proposent d'utiliser un système de coordonnées de type lagrangien, a priori mieux adapté à l'étude des grandes déformations d'une couche de sol. Dans ce système, appelé matériel, la variation de volume d'une couche de sol élémentaire est égale à la variation du volume d'eau interstitielle. L'expression de l'équation de conservation à l'aide de l'indice des vides, e, en fonction du temps et de la cote matérielle $\mathrm{z}$ donne:

$\frac{\partial \mathrm{e}}{\partial \mathrm{t}}+\frac{\gamma_{\mathrm{s}}{ }^{\prime}}{\gamma_{\mathrm{w}}} \cdot \frac{\mathrm{d}\left(\frac{\mathrm{k}}{1+\mathrm{e}}\right)}{\mathrm{de}} \cdot \frac{\partial \mathrm{e}}{\partial \mathrm{z}}+\frac{1}{\gamma_{\mathrm{w}}} \cdot \frac{\partial}{\partial \mathrm{z}}\left[\frac{\mathrm{k}}{1+\mathrm{e}} \cdot \frac{\mathrm{d} \sigma^{\prime}}{\mathrm{de}} \cdot \frac{\partial \mathrm{e}}{\partial \mathrm{z}}\right]=0$ (2) où $\gamma_{\mathrm{w}}$ est le poids volumique de l'eau $\left(9.81 \mathrm{kN} / \mathrm{m}^{3}\right.$ pour l'eau douce), $\gamma_{\mathrm{s}}$ ' est le poids volumique déjaugé des grains $\left(\gamma_{\mathrm{s}}{ }^{\prime}=\gamma_{\mathrm{s}}-\gamma_{\mathrm{w}}\right.$ avec $\gamma_{\mathrm{s}}$ de l'ordre de 23 à $30 \mathrm{kN} / \mathrm{m}^{3}$, selon la composition des solides), $\mathrm{k}$ la perméabilité et $\sigma^{\prime}$ la contrainte effective, fonctions de l'indice des vides seulement. Il a été montré par ailleurs (par exemple, Gallois 1995) que la théorie de Gibson constitue une extension au cas des grandes déformations de la théorie de Terzaghi à laquelle elle se ramène sous les mêmes hypothèses.

\section{Liaison sédimentation-consolidation}

Nous avons mis en évidence, en introduction à cet article, la continuité physique du processus de sédimentation- 
consolidation et souligné l'intérêt d'une approche théorique globale. Dans des travaux récents, dont nous rappelons ici les principales avancées, certains auteurs ont amorcé l'étude de la liaison sédimentation-consolidation.

Been (1981) a établi la correspondance entre la loi de Kynch et la loi de Gibson dans laquelle les contraintes effectives sont négligées. Ce constat permet d'envisager l'utilisation de la loi de Gibson pour la modélisation de la consolidation et de la sédimentation.

Dans ce but, Pane et Schiffman (1985) proposent d'adopter une loi de contrainte effective constamment nulle en sédimentation. Mais cette méthode rend nécessaire la définition d'une frontière entre sédimentation et consolidation. Par ailleurs (Alexis et al. 1993) nous avons montré que la contrainte effective n'apparaissant dans la loi de Gibson que sous forme d'une dérivée par rapport à l'indice des vides, il suffit d'une loi de contrainte effective de forme asymptotique (telle que la contrainte effective et sa dérivée tendent vers 0 aux très grands indices des vides) pour rendre la loi de Gibson applicable à la sédimentation et à la consolidation. Cette approche a le mérite de ne plus distinguer a priori sédimentation et consolidation.

\section{Modélisation continue du processus de sédimentation-consolidation}

La sédimentation-consolidation des sédiments fins est un processus complexe en raison de l'interaction de phénomènes (tels que floculation, ségrégation, érosion ...) dont la prise en compte devra constituer un objectif principal de nouveaux développements théoriques. Afin de permettre de tels développements nous proposons un modèle reposant sur des hypothèses moins restrictives que celles émises par les auteurs cités précédemment.

\section{Hypothèses}

Les extensions de la théorie de Gibson présentent des limites dues à l'utilisation de coordonnées lagrangiennes notamment pour la prise en compte de l'apport de sédiments ou de l'érosion, de la ségrégation, ou encore des caractéristiques de la limite eau-sol. De plus, ces théories supposent le milieu saturé et incompressible, les contraintes effectives fonction seulement de la concentration des solides et les écoulements conformes à la loi de Darcy.

Nous choisissons plutôt d'utiliser un système de coordonnées naturelles composé de deux variables indépendantes: la cote $\mathrm{x}$ selon un axe normé d'origine fixe et orienté vers le haut et le temps t. Nous admettons la compressibilité du fluide et de la structure «solide» (grains, agrégats, flocons ...), en raison, par exemple, de la présence de gaz au sein de l'une et/ou l'autre partie. Nous n'écrirons donc que deux équations de conservation, l'une pour les agrégats, l'autre pour le fluide. Nous supposons enfin que la contrainte effective varie en fonction de l'indice des vides (ou de la concentration) et du temps et que la vitesse de filtration (caractérisant l'écoulement) est fonction quelconque (non nécessairement conforme à la loi de Darcy) de l'indice des vides et du gradient hydraulique. Ces deux dernières hypothèses sont justifiées par nos travaux expérimentaux (Gallois 1995).

\section{Définitions}

Nous allons définir de façon précise les quatre paramètres les plus importants de cette modélisation.

\section{Indice des vides des agrégats}

Lors de la phase de sédimentation notamment, les particules fines s'agglomèrent en flocons ou en agrégats dont la perméabilité peut être considérée comme négligeable devant celle du milieu. On peut donc considérer que le fluide contenu dans un agrégat est fixé pendant toute la durée de son existence. La proportion des constituants (grains et fluide) est donc parfaitement définie par le poids volumique de l'agrégat, $\gamma_{\mathrm{a}}$, et son indice des vides interne, $\mathrm{e}_{\mathrm{a}}$ (rapport du volume de fluide à celui des grains). La formation des agrégats et leur rupture peut ainsi être prise en compte par une loi de variation de $\mathrm{e}_{\mathrm{a}}$.

Indice des vides inter-agrégats

En milieu floculé, la définition habituelle de l'indice des vides (rapport du volume global des vides au volume global des solides) n'est plus adaptée à l'étude de l'écoulement car les vides au sein des agrégats ne sont pas perméables. Nous définissons donc un indice des vides inter-agrégats, $e_{i}$, rapport du volume des vides disponibles pour l'écoulement au volume global des agrégats. Connaissant le poids volumique des solides, $\gamma_{s}$, l'indice des vides classique est lié à l'indice inter-agrégats par la relation:

$e=\left(1+e_{i}\right) \cdot\left(1+e_{a}\right)-1$

\section{Vitesse de filtration}

Ainsi que nous l'avons souligné en introduction, et conformément à notre objectif de modélisation continue de l'ensemble du processus de sédimentation-consolidation, du fait de l'existence simultanée de mouvements d'eau et de solides, la vitesse relative de l'eau par rapport aux grains $\left(\mathrm{v}_{\mathrm{f}}-\mathrm{v}_{\mathrm{a}}\right)$ est un paramètre bien adapté à la description des écoulements. Pour faciliter la mise en équation et par conformité aux travaux de Darcy, nous rapportons cette vitesse relative à la porosité locale disponible pour l'écoulement: $e_{i} /\left(1+e_{i}\right)$. Nous définissons donc la vitesse de filtration $\mathrm{v}_{\mathrm{m}}$, en fonction des vitesses absolues du fluide interstitiel $\mathrm{v}_{\mathrm{f}}$, et des agrégats $\mathrm{v}_{\mathrm{a}}$ (l'indice a remplace l'indice $s$ utilisé pour des grains supposés individualisés et de densité constante):

$\mathrm{v}_{\mathrm{m}}=\frac{\mathrm{e}_{\mathrm{i}}}{1+\mathrm{e}_{\mathrm{i}}} \cdot\left(\mathrm{v}_{\mathrm{f}}-\mathrm{v}_{\mathrm{a}}\right)$

\section{Surpression interstitielle}

Dans un dépôt en équilibre, la pression hydrostatique du fluide en un point est égale au poids par unité de surface de la colonne de fluide située au dessus de celui-ci. Au 
cours du tassement, le fluide interstitiel s'évacue sous l'effet de pressions plus élevées que la pression hydrostatique. Nour appelons surpression interstitielle la différence entre la pression interstitielle totale, $\mathrm{u}_{\mathrm{f}}$, et la pression hydrostatique, $\mathrm{u}_{0}$, soit:

$\mathrm{u}=\mathrm{u}_{\mathrm{f}}-\mathrm{u}_{0}$

\section{Mise en équations}

Nous écrivons successivement, pour le milieu diphasique ainsi défini, les équations d'état, équations de conservation de la matière et équations d'équilibre mécanique. Le système ainsi obtenu est complété par des lois de comportement.

\section{Compressibilité}

Les deux parties en présence (partie fluide et partie liée aux grains) sont supposées compressibles en raison notamment de la présence de gaz. Leurs poids volumiques respectifs $\gamma_{\mathrm{f}}$ et $\gamma_{\mathrm{a}}$ sont donc fonction de la pression locale $\mathrm{u}_{\mathrm{f}}$ :

$\gamma_{\mathrm{f}}=\gamma_{\mathrm{f}}\left(\mathrm{u}_{\mathrm{f}}, \ldots\right)$

$\gamma_{\mathrm{a}}=\gamma_{\mathrm{a}}\left(\mathrm{u}_{\mathrm{f}}, \mathrm{e}_{\mathrm{a}}, \ldots\right)$

\section{Equations de conservation}

En raison de cette compressibilité, les équations de conservation des grains (8) et du fluide (9) portent sur les poids. Nous écrivons ces équations sous une forme générale permettant de prendre en compte les transferts de matière $\delta_{\mathrm{ma}}$ (variation de poids de la phase aggrégative) et $\delta_{\mathrm{mf}}$ (variation de poids de la phase fluide).

$\frac{\partial}{\partial \mathrm{t}}\left(\frac{\gamma_{\mathrm{a}}}{1+\mathrm{e}_{\mathrm{i}}}\right)+\frac{\partial}{\partial \mathrm{x}}\left(\frac{\gamma_{\mathrm{a}} \cdot \mathrm{v}_{\mathrm{a}}}{1+\mathrm{e}_{\mathrm{i}}}\right)=\delta_{\mathrm{ma}}$

$\frac{\partial}{\partial t}\left(\frac{e_{i} \cdot \gamma_{f}}{1+e_{i}}\right)+\frac{\partial}{\partial x}\left(\frac{e_{i} \cdot \gamma_{\mathrm{f}} \cdot v_{f}}{1+e_{i}}\right)=\delta_{m f}$

Transferts de masse

Nous supposons que les transferts de matière entre la partie agrégative et la partie fluide de notre système se réduisent à une expulsion de fluide par les agrégats lors de leur destruction ou (à l'inverse) à la fixation d'une certaine quantité de ce fluide lors de la floculation. Les termes de transfert introduits ci-dessus peuvent donc être considérés comme égaux et exprimés sous la forme d'une variation de poids de fluide dans les agrégats:

$\delta_{\mathrm{ma}}=-\delta_{\mathrm{mf}}=\frac{\partial}{\partial \mathrm{t}}\left(\frac{\mathrm{e}_{\mathrm{a}} \cdot \gamma_{\mathrm{f}}}{1+\mathrm{e}_{\mathrm{a}}}\right)$

\section{Equations d'équilibre}

La contrainte totale, $s$, en un point est égale au poids par unité de surface du matériau susjacent. Cette relation peut être écrite sous forme intégrale ou sous forme locale, en fonction du poids volumique du matériau, $\gamma$, ou des poids volumiques respectifs de chacune des parties:

$\frac{\partial \sigma}{\partial \mathrm{x}}=-\gamma=-\left(\frac{\gamma_{\mathrm{a}}}{1+\mathrm{e}_{\mathrm{i}}}+\frac{\mathrm{e}_{\mathrm{i}} \cdot \gamma_{\mathrm{f}}}{1+\mathrm{e}_{\mathrm{i}}}\right)$
De même, la pression hydrostatique, $\mathrm{u}_{\mathrm{o}}$, est liée au poids volumique du fluide par:

$\frac{\partial u_{0}}{\partial x}=-\gamma_{f}$

\section{Postulat de Terzaghi}

Le postulat de Terzaghi exprime que la contrainte totale en un point du matériau se répartit entièrement en une contrainte sur le fluide (la pression interstitielle $\mathrm{u}_{\mathrm{f}}$ ) et une contrainte inter-agrégats (la contrainte effective $\sigma^{\prime}$ ):

$\sigma=\sigma^{\prime}+\mathrm{u}_{\mathrm{f}}$

\section{Lois de comportement}

Les lois de contrainte effective et d'écoulement au sein d'un matériau sont considérées comme des relations caractéristiques correspondant spécifiquement à ce matériau. Leur connaissance est donc un élément essentiel de la modélisation du comportement d'un sédiment en sédimentation-consolidation. Nous avons effectué par ailleurs (Alexis et al. 1993; Gallois 1995) une analyse bibliographique et théorique des différentes formes de lois proposées dans des travaux antérieurs (Koppula et Morgenstern 1982; Gibson et al.1981; Sanchez 1992; Richardson et Zaki 1954).

Par leur forme univariable, $\sigma^{\prime}(\mathrm{e})$, les lois de contraintes effectives nous paraissent mal adaptées à la prise en compte de phénomènes tels que le fluage dont l'importance vis à vis du processus de sédimentation-consolidation, très lent, ne peut pas être négligée a priori. Nous introduisons donc le temps comme seconde variable et admettons comme loi de contrainte effective:

$\sigma^{\prime}=\sigma^{\prime}\left(\mathrm{e}_{\mathrm{i}}, \mathrm{t}\right)$

Dans un même souci de généralisation, à l'application directe de la loi de Darcy $\left(\mathrm{v}_{\mathrm{m}}=\mathrm{k}\left(\mathrm{e}_{\mathrm{i}}\right) \cdot \mathrm{i}\right)$, où la perméabilité $\mathrm{k}$ dépendrait seulement de l'indice des vides $\mathrm{e}_{\mathrm{i}}$, nous préférons admettre, comme loi d'écoulement, la forme générale fonction de l'indice des vides et du gradient de surpressions:

$\mathrm{v}_{\mathrm{m}}=\mathrm{v}_{\mathrm{m}}\left(\mathrm{e}_{\mathrm{i}}, \partial \mathrm{u} / \partial \mathrm{x}\right)$

Nos travaux expérimentaux (Gallois 1995) ont, par exemple, mis en évidence un gradient hydraulique dit critique, $\mathrm{i}_{\mathrm{c}}\left(\mathrm{e}_{\mathrm{i}}\right)$, en-deça duquel l'écoulement est négligeable $\left(v_{m}=0\right)$. Pour $i=1 / \gamma_{w} \cdot \partial u / \partial x>i_{c}$, la loi d'écoulement prendrait alors la forme:

$v_{m}=k^{\prime}\left(e_{i}\right) \cdot\left[i-i_{c}\left(e_{i}\right)\right]$

où $\mathrm{k}^{\prime}$, comme la perméabilité, a la dimension d'une vitesse.

\section{Résolution}

Nous obtenons un système de 13 équations [(3) à (15)] à 13 inconnues $\left(\mathrm{e}, \mathrm{e}_{\mathrm{i}}, \mathrm{v}_{\mathrm{m}}, \mathrm{v}_{\mathrm{f}}, \mathrm{v}_{\mathrm{a}}, \mathrm{u}, \mathrm{u}_{\mathrm{f}}, \mathrm{u}_{\mathrm{o}}, \gamma_{\mathrm{f}}, \gamma_{\mathrm{a}}, \delta_{\mathrm{m}}, \sigma, \sigma^{\prime}\right)$, dans lequel les expressions des lois de compressibilité [(6) et (7)], et de comportement [(14) et (15)] nécessitent une approche particulière. Une fois ces lois définies, le système 
peut être résolu numériquement, par une méthode de différences finies par exemple.

\section{Procédure de validation}

\section{Conditions limites}

La généralité de notre modèle permet son application à un grand nombre de cas de sédimentation et/ou de consolidation caractérisés chacun par des conditions initiales et des conditions aux frontières du domaine: sédimentationconsolidation sous l'eau (Fig. 1), avec niveau d'eau variable (prise en compte de la marée, de la houle, ...), consolidation de dépôts terrestres de vase sous poids propre, consolidation de couches d'argiles molles sous charge, essais œdométriques.

\section{Conditions initiales}

Le modèle que nous proposons met en jeu deux équations (de conservation) différentielles du premier ordre en t. Sa résolution suppose donc, pour l'instant initial, la connaissance en tout point du domaine de deux des grandeurs étudiées. En pratique, on pourra, par exemple, simuler la sédimentation-consolidation d'une couche initialement homogène et en définissant l'indice des vides et une vitesse initiale (généralement nulle).

\section{Conditions aux frontières}

De même l'utilisation de cinq équations différentielles du premier ordre en $\mathrm{x}$ impose la définition de cinq conditions aux limites du dépôt. Les conditions de pression à la surface et les conditions de drainage au fond du dépôt fournissent les valeurs au frontières nécessaires.

\section{Validation}

Nous effectuons la validation en deux étapes:

1. Liaison avec la théorie de Gibson.

2. Confrontation expérimentation-simulation.

Liaison avec la théorie de Gibson

Nous appliquons à notre modèle les hypothèses de Gibson. Les grains étant incompressibles et la floculation non prise en compte, $\gamma_{\mathrm{a}}$ est constant. Le fluide étant incompressible, $\gamma_{\mathrm{f}}=\gamma_{\mathrm{w}}$. En l'absence de transfert de masse entre phases, les équations de conservation s'écrivent donc:

$\frac{\partial}{\partial \mathrm{t}}\left(\frac{1}{1+\mathrm{e}_{\mathrm{i}}}\right)+\frac{\partial}{\partial \mathrm{x}}\left(\frac{\mathrm{v}_{\mathrm{a}}}{1+\mathrm{e}_{\mathrm{i}}}\right)=0$

$\frac{\partial}{\partial t}\left(\frac{e_{i}}{1+e_{i}}\right)+\frac{\partial}{\partial x}\left(\frac{e_{i} \cdot v_{f}}{1+e_{i}}\right)=0$

L'équation (16) s'écrit aussi:

$\frac{\partial \mathrm{e}_{\mathrm{i}}}{\partial \mathrm{t}}-\left(1+\mathrm{e}_{\mathrm{i}}\right)^{2} \cdot \frac{\partial}{\partial \mathrm{x}}\left(\frac{\mathrm{v}_{\mathrm{a}}}{1+\mathrm{e}_{\mathrm{i}}}\right)=0$

et le somme de (16) et (17) donne (*en supposant le fond du déplôt imperméable): $\frac{\partial v_{m}}{\partial x}+\frac{\partial v_{s}}{\partial x}=0$ soit ${ }^{*} v_{m}=-v_{s}$

Enfin, en choisissant la loi de Darcy comme loi d'écoulement, et en supposant que la perméabilité réduite $\left(\mathrm{k}_{\mathrm{r}}=\mathrm{k} /\left(1+\mathrm{e}_{\mathrm{i}}\right)\right)$ et les contraintes effectives dépendent seulement de l'indice des vides $e_{i}$, on obtient l'équation suivante:

$$
\begin{aligned}
& \frac{\partial e_{i}}{\partial t}-\frac{\gamma_{a}^{\prime}}{\gamma_{w}}\left(1+e_{i}\right) \frac{d\left(\frac{k_{f}}{1+e_{i}}\right)}{d e} \cdot \frac{\partial e_{i}}{\partial x}+\frac{\left(1+e_{i}\right)^{2}}{\gamma_{w}} \\
& \cdot \frac{\partial}{\partial x}\left[k_{f} \frac{d \sigma^{\prime}}{d e_{i}} \cdot \frac{\partial e_{i}}{\partial x}\right]=0
\end{aligned}
$$

Cette formulation en coordonnées naturelles est analogue à l'équation en coordonnées matérielles de Gibson (2).

Une telle simplification est utile à ce stade pour valider provisoirement notre modèle, dans l'attente d'une nouvelle approche des lois (6), (7), (14) et (15), qui permettra d'utiliser pleinement la généralité de notre théorie.

\section{Confrontation expérimentation-simulation}

Been (1981) a étudié expérimentalement la sédimentation et le tassement sous poids propre d'échantillons de vase placés dans des colonnes verticales en laboratoire. Des mesures de densité effectuées en différents points au cours $\mathrm{du}$ processus de sédimentation-consolidation lui ont permis d'établir des profils de densité du matériau. L'expérience $\mathrm{n}^{\circ} 15$ de Been est une de celle pour lesquelles les résultats sont les plus nombreux. Elle étudie pendant 35 jours l'évolution d'un dépôt initialement homogène (densité 1.146) de hauteur $67 \mathrm{~cm}$ (Fig. 2).

Pour valider notre modèle, nous nous proposons de reproduire par le calcul cette expérience de Been. Des lois de comportement simples à une seule variable, $\sigma^{\prime}=\mathrm{a} \cdot(\mathrm{e}-\mathrm{b})^{-\mathrm{c}}$ et $\mathrm{k}_{\mathrm{r}}=\mathrm{a} \cdot(\mathrm{e} /(1+\mathrm{e}))^{\mathrm{b}}$ (Gallois 1995), ont été ajustées aux valeurs expérimentales de perméabilité et de contrainte effective calculées par Been.

La confrontation des profils expérimentaux et calculés (Fig. 3) montrent une très bonne correspondance des densités atteintes au fond du dépôt ainsi que de la vitesse de chute de l'interface eau-sédiment. Les principales différences entre profils expérimentaux et calculés sont liées à l'existence, sur les profils de Been, de très forts gradients de densité à l'interface eau-sédiment (qui est donc parfaitement nette) ainsi qu'au fond, dans les premières heures de consolidation. Notre modèle donne des gradients moins forts et des transitions plus progressives entre les différents milieux, en accord avec nos propres observations (Gallois 1995).

\section{Conclusion}

Jusqu'alors, les théories de la sédimentation (modélisée par les hydrauliciens) et de la consolidation (étudiée par les géotechniciens) étaient disjointes. Dans cet article nous 


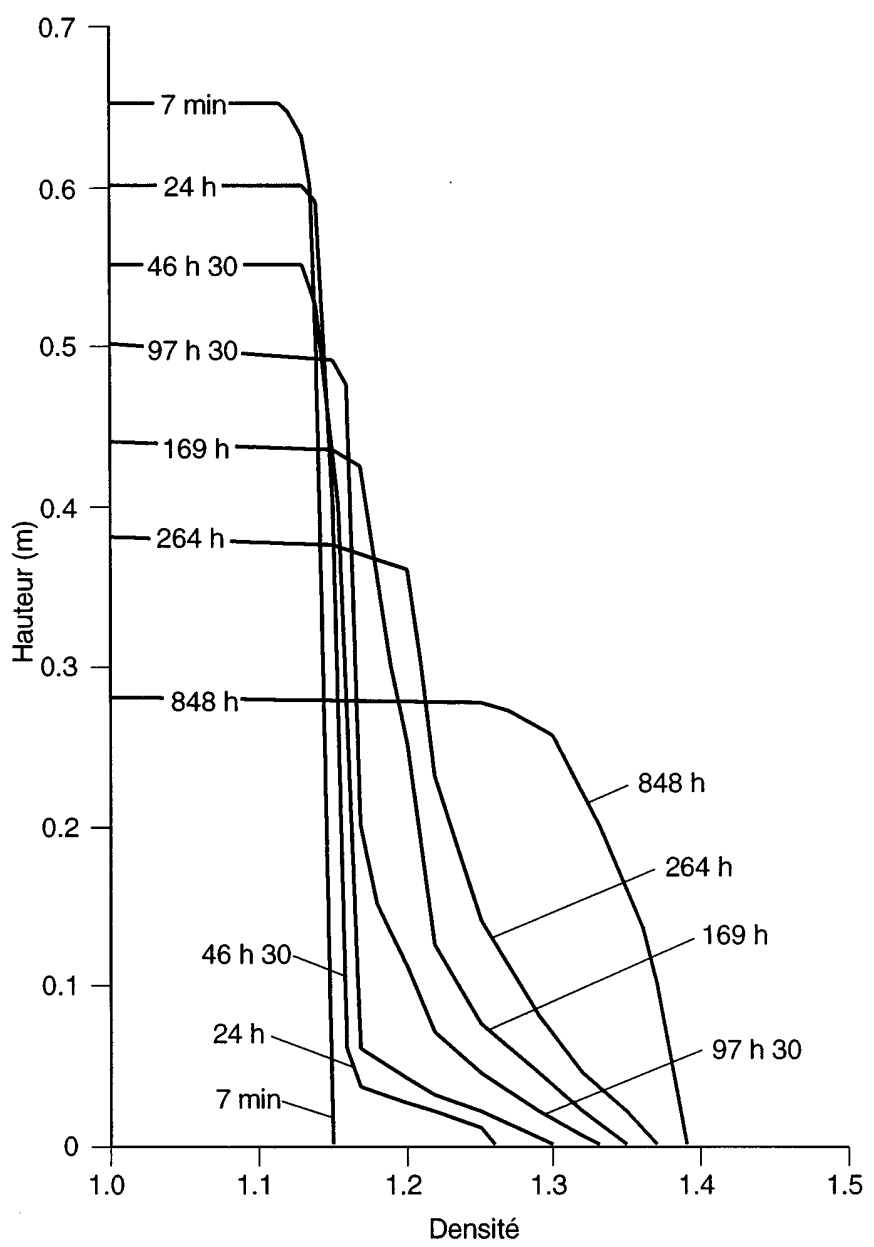

Fig. 2

Profils de densité obtenus par Been (exp. $\left.n^{\circ} 15,1981\right)$ d'un essai de sédimentation-consolidation en laboratoire

Fig. 2

Been's density profiles (exp. no. 15, 1981) from laboratory sedimentation-consolidation tests

proposons une modélisation qui intègre et prolonge les travaux antérieurs par l'unification des théories de la sédimentation et de la consolidation.

La généralisation de la modélisation que nous proposons est d'ordre spatial, mathématique et phénoménologique. En effet, par rapport aux modèles antérieurs, elle se caractérise par:

1. Un domaine d'application plus large.

2. Des choix mathématiques moins restrictifs.

3. Des lois de comportement plus ouvertes.

et cette modélisation rend possible la prise en compte de nombreux aspects, négligés jusqu'alors, de la complexité $\mathrm{du}$ matériau et de son environnement (non saturation, émersion ou immersion, fluage, sédimentation des flocons, écoulements non conformes à la loi de Darcy, apports en suspension et érosion, ...).

Dans un objectif d'application à l'étude de la dynamique sédimentaire des vases en environnement estuarien, nous avons ainsi constitué une modélisation du comportement

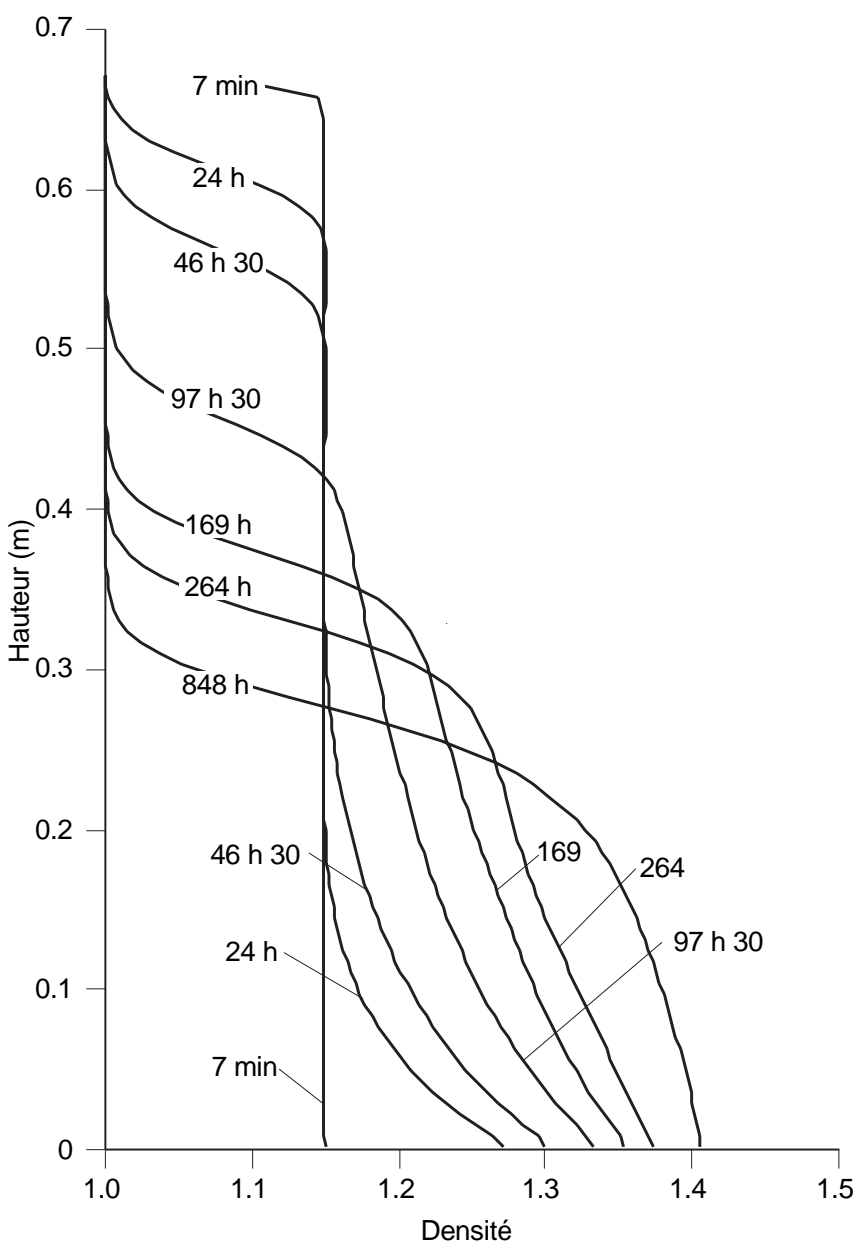

Fig. 3

Profils de densité calculés par notre modèle en simulation de l'expérience $\mathrm{n}^{\circ} 15$ de Been (1981)

Fig. 3

Density profiles computed by our model simulating Been's no. 15 experiment (1981)

d'un milieu poreux dans son passage de l'état dispersé à l'état structuré.

\section{References}

Alexis A, Thomas P, Gallois S (1993) Tassement des sédiments cohésifs. Rapport final de contrat MAST 1, E.D.F./ IFREMER/I.U.T. de Saint-Nazaire, janv. 1993

BEEN K (1981) Stress strain behaviour of a cohesive sediment deposited under water. $\mathrm{PhD}$ thesis, Oxford University, Oxford Gallois S (1995) Modélisation de la sédimentation-consolidation et expérimentations sur les vases estuariennes. Thèse de doctorat, Ecole Centrale/Université de Nantes, Nantes

Gibson RE, England GL, Hussey MJL (1967) The theory of onedimensional consolidation of saturated clays. I. Finite nonlinear consolidation of thin homogeneous layers. Géotechnique $17: 261-273$ 
Gibson RE, Schiffman RL, Cargill KW (1981) The theory of one-dimensional consolidation of saturated clays. II. Finite non-linear consolidation of thick homogeneous layers, Can Geotech J 18:280-293

Koppula SD, Morgenstern NR (1982) On the consolidation of sedimenting clays. Can Geotech J, 19:260-268

KynCH GJ (1952) A theory of sedimentation. Trans Faraday Soc 48:166-176

Pane V, Schiffman RL (1985) A note on sedimentation and consolidation. Géotechnique 35:69-72
RICHARDSON JF, ZAKI WN (1954) Sedimentation and fluidisation I. Trans Inst Chem Eng 32:35-53

SANCHEZ M (1992) Modélisation dans un estuaire à marée: rôle $\mathrm{du}$ bouchon vaseux dans la tenue des sols sous-marins. Thèse de doctorat, Université de Nantes, Nantes

Terzaghi K (1942) Theoretical soil mechanics. Wiley, New York

Toorman EA (1992) Modelling of fluid mud and consolidation. $\mathrm{PhD}$ thesis, Katholieke Universiteit Leuwen, Leuwen 